\title{
Bell non-locality in macroscopic systems
}

\author{
B.J. Dalton ${ }^{1,2, a}$ \\ ${ }^{1}$ Centre for Quantum and Optical Science, Swinburne University of Technology, \\ Melbourne, Victoria 3122, Australia \\ ${ }^{2}$ School of Physics and Astronomy, University of Glasgow, Glasgow G12 8QQ, UK
}

Received 12 April 2018 / Received in final form 18 July 2018

Published online 13 February 2019

\begin{abstract}
The categorization of quantum states for composite systems as either separable or entangled, or alternatively as Bell local or Bell non-local states based on local hidden variable theory is outlined, focusing on simple bipartite systems. The significance of states demonstrating Bell non-locality for settling the long standing controversy between the Copenhagen interpretation of the quantum measurement process involving "collapse of the wave-function" and the alternative interpretation based on pre-existing hidden variables is emphasized. Although experiments demonstrating violations of Bell locality in microscopic systems have now been carried out, there is current interest in finding Bell non-locality in quantum systems on a macroscopic scale, since this is a regime where a classical hidden variable theory might still apply. Progress towards finding macroscopic quantum states that violate Bell inequalities is reviewed. A new test for Bell non-locality that applies when the sub-system measured quantities are spin components with large outcomes is described, and applied to four mode systems of identical massive bosons in Bose-Einstein condensates.
\end{abstract}

\section{Copenhagen interpretation and EPR paradox}

To Einstein [1], the Copenhagen quantum interpretation of what happens in bipartite systems when we first measure an observable $\Omega_{A}$ in one sub-system $A$ with outcome $\alpha$, and then immediately measure an observable $\Omega_{B}$ in a second well-separated sub-system $B$ with outcome $\beta$ seemed counter-intuitive - implying "instantaneous action at a distance" during the two-step measurement process. This is known since the 1930 s as the EPR paradox. According to the Copenhagen interpretation, if the quantum state resulting from preparation process $c$ is $\widehat{\rho}$, then after the first measurement the quantum state changes to the conditioned state $\widehat{\rho}_{\text {cond }}\left(\alpha \mid \Omega_{A}, \rho\right)=$ $\left(\widehat{\Pi}_{\alpha}^{A} \otimes \widehat{1}^{B}\right) \widehat{\rho}\left(\widehat{\Pi}_{\alpha}^{A} \otimes \widehat{1}^{B}\right) / P\left(\alpha \mid \Omega_{A}, \rho\right)$, where $\widehat{\Pi}_{\alpha}^{A}$ is the projector onto the eigenvector space of $\widehat{\Omega}_{A}$ with eigenvalue $\alpha$, and $P\left(\alpha \mid \Omega_{A}, \rho\right)=\operatorname{Tr}\left(\left(\widehat{\Pi}_{\alpha}^{A} \otimes \widehat{1}^{B}\right) \widehat{\rho}\right)$ is the probability for state $\widehat{\rho}$ that measurement of $\Omega_{A}$ leads to outcome $\alpha$. The density operator is normalized as $\operatorname{Tr} \widehat{\rho}=1$. In general, the reduced density operator $\widehat{\rho}_{B}=\operatorname{Tr}_{A} \widehat{\rho}$ describing the original state for sub-system $B$ would be instantly changed to a different state

a e-mail: bdalton@swin. edu.au 
$-\operatorname{Tr}_{A}\left(\widehat{\rho}_{\text {cond }}\left(\alpha \mid \Omega_{A}, \rho\right)\right)$, even though no signal would have had time to travel between the two well-separated sub-systems. This effect is referred to as steering [2]. Of course if $\Omega_{A}$ was immediately measured a second time it is easy to show that the outcome $\alpha$ would occur with probability 1 . For the Copenhagenist, the quantum state $\widehat{\rho}$ is not itself a real object, but only a means of determining the probabilities of the outcomes of measuring observables - the outcomes being the real objects which are created by the measurement process on the prepared quantum state. That the quantum state changes as a result of the measurement of $\Omega_{A}$ with outcome $\alpha$, merely signifies the probability changing from $\operatorname{Tr}\left(\left(\widehat{\Pi}_{\alpha}^{A} \otimes \widehat{1}^{B}\right) \widehat{\rho}\right) \neq 1$ for the original preparation process to $\operatorname{Tr}\left(\left(\widehat{\Pi}_{\alpha}^{A} \otimes \widehat{1}^{B}\right) \widehat{\rho}_{\text {cond }}\left(\alpha \mid \Omega_{A}, \rho\right)\right)=1$ for a new preparation process in which the second part involves measuring $\Omega_{A}$ with outcome $\alpha$. If we now measure the second sub-system observable $\Omega_{B}$ the conditional probability $P\left(\beta\left|\Omega_{B}\right||\alpha| \Omega_{A}, \rho\right)$ for outcome $\beta$, given that measurement of $\Omega_{A}$ in the first sub-system $A$ resulted in outcome $\alpha$, will now be determined from the conditioned state as $P\left(\beta \mid \Omega_{B}, \rho_{\text {cond }}\left(\alpha \mid \Omega_{A}, \rho\right)\right)=$ $\left.\operatorname{Tr}\left(\left(\widehat{1}^{A} \otimes \widehat{\Pi}_{\beta}^{B}\right) \widehat{\rho}_{\text {cond }}\left(\alpha \mid \Omega_{A}, \rho\right)\right)=\operatorname{Tr}\left(\widehat{\Pi}_{\alpha}^{A} \otimes \widehat{\Pi}_{\beta}^{B}\right) \widehat{\rho}\right) / P\left(\alpha \mid \Omega_{A}, \rho\right)$. In general this will be different from the probability $P\left(\beta \mid \Omega_{B}, \rho\right)=\operatorname{Tr}\left(\left(\widehat{1}^{A} \otimes \widehat{\Pi}_{\beta}^{B}\right) \widehat{\rho}\right)=\operatorname{Tr}_{B}\left(\left(\widehat{\Pi}_{\beta}^{B}\right) \widehat{\rho}_{B}\right)$ of outcome $\beta$ resulting from measurement of observable $\Omega_{B}$ for the original state $\widehat{\rho}$ when no measurement of $\Omega_{A}$ is made. However, using Bayes' theorem the joint probability for outcomes $\alpha$ for $\Omega_{A}$ and $\beta$ for $\Omega_{B}$ can be determined as $P\left(\alpha, \beta \mid \Omega_{A}, \Omega_{B}, \rho\right)=$ $P\left(\beta \mid \Omega_{B}, \rho_{\text {cond }}\left(\alpha \mid \Omega_{A}, \rho\right)\right) \times P\left(\alpha \mid \Omega_{A}, \rho\right)=\operatorname{Tr}\left(\left(\widehat{\Pi}_{\alpha}^{A} \otimes \widehat{\Pi}_{\beta}^{B}\right) \widehat{\rho}\right)$. This is the standard Copenhagen expression for the joint measurement probability for the measurement of the two observables in the separated sub-systems if the measurements had been made on the original state $\widehat{\rho}$ totally independently of each other and in no particular order. As far as we know, the predictions based on Copenhagen version of quantum theory are always in accord with experiment. But to Einstein and others, the Copenhagen theoretical picture was philosophically unsatisfactory. So the question arose is it really necessary to invoke the Copenhagen picture involving the instantaneous change to the quantum state as a result of the first measurement (the "collapse of the wave function") to describe what happens, or is there a simpler picture based on classical probability theory - and involving what we now refer to as hidden variables - that could also account for all the quantum theory probability predictions?

\subsection{Hidden variable theory and Bell non-locality}

The EPR paradox remained an unresolved issue for many years. However in the 1960s Bell [3] proposed a quantitative version of a general hidden variable theory which led to certain inequalities (the Bell inequalities) involving measurable quantities (such as the mean values for the measurement outcomes of sub-system observables) which could also be calculated using standard quantum theory. This suggested that experimental tests could be carried out to compare the results from quantum theory with those from hidden variable theory. In hidden variable theory the preparation process $c$ determines a probabilistic distribution $P(\lambda, c)$ of hidden variables $\lambda$. The detailed nature of the hidden variables is irrelevant, but we require $\sum_{\lambda} P(\lambda, c)=1$. The hidden variables may change with time in accordance with as yet unspecified dynamical equations, and thus would determine the system's underlying evolution. Here, we just focus on measurements carried out at some particular time and the hidden variables $\lambda$ are those that apply at the time of measurement, though they are still determined from the original preparation process. In accordance with the ideas of classical physics, it may be assumed that measurements of observables $\Omega$ can be carried out leading to a possible outcome $\alpha$ without any significant perturbation of the underlying dynamics. For bipartite systems, in each of the two sub-systems the 
hidden variables in a local hidden variable theory (LHVT) specify separate classical probabilities $P\left(\alpha \mid \Omega_{A}, \lambda, c\right)$ and $P\left(\beta \mid \Omega_{B}, \lambda, c\right)$ that measurement of observables $\Omega_{A}$, $\Omega_{B}$ in the respective sub-systems $A, B$ leads to outcomes $\alpha, \beta$. The joint probability for outcomes $\alpha$ for $\Omega_{A}$ and $\beta$ for $\Omega_{B}$ is then determined in accord with classical probability theory as $P\left(\alpha, \beta \mid \Omega_{A}, \Omega_{B}, c\right)=\sum_{\lambda} P(\lambda, c) P\left(\alpha \mid \Omega_{A}, \lambda, c\right) P\left(\beta \mid \Omega_{B}, \lambda, c\right)$, and the probability for outcome $\alpha$ for measuring $\Omega_{A}$ alone would be given by $P\left(\alpha \mid \Omega_{A}, c\right)=\sum_{\lambda} P(\lambda, c) P\left(\alpha \mid \Omega_{A}, \lambda, c\right)$. This gives the conditional probability for outcome $\beta$, given that measurement of $\Omega_{A}$ in the first sub-system $A$ resulted in outcome $\alpha$ as $P\left(\beta\left|\Omega_{B}\right||\alpha| \Omega_{A}, c\right)=\sum_{\lambda} P(\lambda, c) P\left(\alpha \mid \Omega_{A}, \lambda, c\right) P\left(\beta \mid \Omega_{B}, \lambda, c\right) /$ $\sum_{\lambda} P(\lambda, c) P\left(\alpha \mid \Omega_{A}, \lambda, c\right)$. These expressions may be compared to those from quantum theory. As LHVT theory is intended to underlie quantum theory, the point is that both the joint probability $P\left(\alpha, \beta \mid \Omega_{A}, \Omega_{B}, c\right)$ and single probabilities such as $P\left(\alpha \mid \Omega_{A}, c\right)$ can be determined from the LHVT probabilities $P(\lambda, c), P\left(\alpha \mid \Omega_{A}, \lambda, c\right)$ and $P\left(\beta \mid \Omega_{B}, \lambda, c\right)$ without requiring a knowledge of the system density operator $\hat{\rho}$. States that can be described via LHVT are referred to as Bell local - those that cannot be so described are Bell non-local. However, apart from the differing forms of the probability expressions, there is a fundamental difference in the description of what happens in the measurement process. In hidden variable theory the hidden variables are determined (at least probabilisticaly) in the preparation process and are carried over to both sub-systems irrespective of how well they are separated. They then determine the probabilities for the outcomes $\alpha, \beta$ of measurements for $\Omega_{A}$ and $\Omega_{B}$ on the two sub-systems. As we are considering measurements at the same time, in local hidden variable theory the outcome of measurement on one sub-system could not affect that for the other sub-system. Unlike the Copenhagen theory change to the quantum state as a result of first measuring $\Omega_{A}$, no instantaneous changes to the hidden variables is invoked, certainly no change dependent on the outcome $\alpha$. Hence, if an experiment could be carried out whose results are in accord with quantum theory but not in accord with this general hidden variable theory, the interpretation that quantum theory is under-pinned by a classical probability theory involving hidden variables would have to be rejected. As quantum theory has been confirmed in a wide range of other experimental situations it would be reasonable to accept its validity (leaving aside the physics of black holes etc.). This does not necessarily imply though that the Copenhagen interpretation of what happens in the measurement process would have to be accepted without further discussion, since other interpretations of quantum theory exist such as the many-worlds [4] or the Bohmian nonlocal realistic [5] interpretations. As these are just different interpretations of quantum theory no experimental test rules these out. However, the many-worlds interpretation invokes the idea that every possible measured outcome occurs with some probability in a separate noncommunicating world, and that separate worlds are created whenever a measurement is made. Philosophically, this interpretation fails the test of simplicity, which favours the Copenhagen interpretation based on a single ongoing probabilistic world. Similar considerations apply to the complicated Bohmian approach, which in its simplest version involves deterministic particle positions, whose dynamical evolution depends on the wave-function as determined from the time-dependent Schrodinger equation, but which can account for experimental results if Born's probability rule is assumed. Thus, if Bell non-local states could be found this would resolve the philosophical issue of what happens in the measurement process in favour of the Copenhagen interpretation if Occum's razor rules out these alternative interpretations. There would therefore be quantum states with correlations for the joint measurement outcomes in separated sub-systems as given by the quantum expression, which are not accounted for via the classical correlations that apply to the hidden variable theory expression. Such quantum correlations are referred to as Bell correlations. 


\subsection{Quantum and hidden variable theory predictions}

Comparisons between the Copenhagen quantum and local hidden variable theory predictions can be made based on Bell inequalities involving the mean values of the measurement results as well as those based directly on the joint measurement probabilities. The quantum theory and the LHVT expressions for the probabilities of joint measurement outcomes $\alpha, \beta$ for $\Omega_{A}, \Omega_{B}$ are

$$
\begin{aligned}
P\left(\alpha, \beta \mid \Omega_{A}, \Omega_{B}, \rho\right)_{Q} & =\operatorname{Tr}\left(\left(\widehat{\Pi}_{\alpha}^{A} \otimes \widehat{\Pi}_{\beta}^{B}\right) \widehat{\rho}\right) \\
P\left(\alpha, \beta \mid \Omega_{A}, \Omega_{B}, c\right)_{L H V T} & =\sum_{\lambda} P(\lambda, c) P\left(\alpha \mid \Omega_{A}, \lambda, c\right) P\left(\beta \mid \Omega_{B}, \lambda, c\right)
\end{aligned}
$$

and we then find that the quantum theory and LHVT expressions for the mean values of the joint measurement outcomes for $\Omega_{A}, \Omega_{B}$ are

$$
\begin{aligned}
\left\langle\Omega_{A} \otimes \Omega_{B}\right\rangle_{Q} & =\operatorname{Tr}\left(\widehat{\Omega}_{A} \otimes \widehat{\Omega}_{B}\right) \widehat{\rho} \\
\left\langle\Omega_{A} \otimes \Omega_{B}\right\rangle_{L H V T} & =\sum_{\lambda} P(\lambda, c)\left\langle\Omega_{A}(\lambda, c)\right\rangle\left\langle\Omega_{B}(\lambda, c)\right\rangle
\end{aligned}
$$

where $\left\langle\Omega_{A}(\lambda, c)\right\rangle=\sum_{\alpha} \alpha P\left(\alpha \mid \Omega_{A}, \lambda, c\right)$ is the hidden variable theory mean value for measurement of $\Omega_{A}$ when the hidden variables are $\lambda$, with a similar result for $\left\langle\Omega_{B}(\lambda, c)\right\rangle$. In addition, comparisons can be made based on measurement outcomes over restricted ranges. For example if both $\alpha$ and $\beta$ were restricted to be positive, then the quantum and LHVT expressions for the joint probabilities of these positive measurement outcomes are

$$
\begin{aligned}
P\left(+,+\mid \Omega_{A}, \Omega_{B}, \rho\right)_{Q} & =\operatorname{Tr}\left(\left(\widehat{\Pi}_{+}^{A} \otimes \widehat{\Pi}_{+}^{B}\right) \widehat{\rho}\right) \\
P\left(+,+\mid \Omega_{A}, \Omega_{B}, c\right)_{L H V T} & =\sum_{\lambda} P(\lambda, c) P\left(+\mid \Omega_{A}, \lambda, c\right) P\left(+\mid \Omega_{B}, \lambda, c\right)
\end{aligned}
$$

where $\widehat{\Pi}_{+}^{A}=\sum_{\alpha>0} \widehat{\Pi}_{\alpha}^{A}$ and $P\left(+\mid \Omega_{A}, \lambda, c\right)=\sum_{\alpha>0} P\left(\alpha \mid \Omega_{A}, \lambda, c\right)$ are projectors and LHVT probabilities for positive outcomes for the measurement of $\Omega_{A}$, with similar expressions for $\Omega_{B}$. Although for simplicity the preceding discussion has focused on bipartite systems, its generalisation to multipartite systems is straight-forward.

Note that if the Heisenberg uncertainty principle is to be satisfied in LHVT for the case of non-commuting observables, extra contraints would be required for the subsystem probabilities. Thus for non-commuting quantum operators where $\left[\widehat{\Omega}_{A 1}, \widehat{\Omega}_{A 2}\right]=$ $i \widehat{M}$, the corresponding LHVT probabilities $P\left(\alpha_{1} \mid \Omega_{A 1}, \lambda, c\right), P\left(\alpha_{2} \mid \Omega_{A 2}, \lambda, c\right)$ must lead to the required condition on the LHVT standard deviations, namely $\Delta \Omega_{A 1} \times \Delta \Omega_{A 1} \geq$ $|\langle M\rangle| / 2$, where $\left(\Delta \Omega_{A 1}\right)^{2}=\sum_{\lambda} \sum_{\alpha_{1}} P(\lambda, c) P\left(\alpha_{1} \mid \Omega_{A 1}, \lambda, c\right)\left(\alpha_{1}-\left\langle\Omega_{A 1}\right\rangle\right)^{2},\left\langle\Omega_{A 1}\right\rangle=$ $\sum_{\lambda} \sum_{\alpha_{1}} P(\lambda, c) P\left(\alpha_{1} \mid \Omega_{A 1}, \lambda, c\right) \alpha_{1}$, etc.

\section{Categorizing bipartite states}

\subsection{Local hidden states - EPR steering and Bell non-locality}

As explained below, the LHVT sub-system probabilities of measurement outcomes for sub-system observables may also be given by quantum expressions involving density operators for the separate sub-systems (and not determined from the overall system 
density operator), and that the preparation process may also determine probabilities for particular sub-system quantum density operators to apply. This involves the concept of local hidden states, which arose first in the case of separable states. Even for the simple case of bipartite systems, this leads to three different categories of Bell local states, together with a fourth category in which LHVT does not apply.

A first question is whether the results for any quantum states describing two subsystems can be also described by local hidden variable theory. One whole class of states that can be so-described are the separable states [6], where the density operator is of the form $\widehat{\rho}_{\text {sep }}=\sum_{R} P_{R} \widehat{\rho}_{R}^{A} \otimes \widehat{\rho}_{R}^{B}$. Here the preparation process involves preparing each separate sub-system in states $\widehat{\rho}_{R}^{A}$ and $\widehat{\rho}_{R}^{B}$, where $P_{R}$ is the probability that a particular choice $R$ has been made. Note that for separable states the reduced density operator for each sub-system $C$ is given by $\widehat{\rho}_{C}=\sum_{R} P_{R} \widehat{\rho}_{R}^{C}$, which in general differs from the sub-system states $\widehat{\rho}_{R}^{C}$. States where $\widehat{\rho} \neq \widehat{\rho}_{\text {sep }}$ are the non-separable or entangled states. For separable states the quantum joint probability is given by $P\left(\alpha, \beta \mid \Omega_{A}, \Omega_{B}, \rho_{\text {sep }}\right)=\sum_{R} P_{R} P\left(\alpha \mid \Omega_{A}, R\right) P\left(\beta \mid \Omega_{B}, R\right)$ where $P\left(\alpha \mid \Omega_{A}, R\right)=\operatorname{Tr}_{A}\left(\left(\widehat{\Pi}_{\alpha}^{A}\right) \widehat{\rho}_{R}^{A}\right)$ and $P\left(\beta \mid \Omega_{B}, R\right)=\operatorname{Tr}_{B}\left(\left(\widehat{\Pi}_{\beta}^{B}\right) \widehat{\rho}_{R}^{B}\right)$ are probabilities for the separate sub-system measurement outcomes, which are given by quantum theory expressions. However, these results are of the same form as in local hidden variable theory, with the choice $R$ being regarded as a hidden variable and with $P_{R} \rightarrow P(\lambda, c)$, $P\left(\alpha \mid \Omega_{A}, R\right) \rightarrow P\left(\alpha \mid \Omega_{A}, \lambda, c\right)$, etc. So as the separable states can all be given a local hidden variable theory interpretation, it follows that any state that cannot be so interpreted must be an entangled state. However, Werner [6] showed that there were some entangled states that could be interpreted in terms of local hidden variable theory. Particular examples were the so-called Werner states [6], which are mixed states specified by a single parameter and involve two sub-systems with equal dimensionality. This means that the division of quantum states into separable or entangled does not coincide with their division into Bell local and Bell non-local.

Wiseman et al. [7] introduced the idea of a so-called local hidden quantum state (LHS) which applied when a particular sub-system $A$ was also associated with a quantum density operator $\widehat{\rho}^{A}(\lambda, c)$ specified by the hidden variables $\lambda$, and which determines the LHVT probability $P\left(\alpha \mid \Omega_{A}, \lambda, c\right)$. The separable states are characterized by both sub-systems being associated with a local hidden quantum state, and are examples of quantum states that can be also described by LHVT (and referred to as Category 1 states). Within local hidden variable theory we could also have the situation where only one of the two sub-systems ( $B$ say), is associated with a local hidden quantum state $\widehat{\rho}^{B}(\lambda, c)$ from which the probability is determined as $P\left(\beta \mid \Omega_{B}, \lambda, c\right)=\operatorname{Tr}_{B}\left(\widehat{\Pi}_{\beta}^{B} \widehat{\rho}^{B}(\lambda, c)\right)$, whilst for the other sub-system $A$ the probability $P\left(\alpha \mid \Omega_{A}, \lambda, c\right)$ is not determined from a local hidden state (referred to as Category 2 states). Another Bell local situation is where neither sub-system is associated with a local hidden quantum state (referred to as Category 3 states). Both these last two situations still involve entangled quantum states, whilst also being described by local hidden variable theory. States where there are no local hidden states are referred to as EPR steerable states [7]. They allow for the possibility of choosing the measurement for observable $\Omega_{A}$ to steer sub-system $B$ such that the outcome for measuring $\Omega_{B}$ can be chosen in advance. The EPR steerable states are all entangled, and include those that are Bell non-local as well as some that are Bell local and entangled. They are said to exhibit EPR correlations. Bell non-local states (where the LHVT expression for the joint probability is not valid at all - will be referred to as Category 4 states, and exhibit the strongest form of correlation between the two sub-systems. To find whether a state is Bell non-local requires showing that a Bell inequality - 


\begin{tabular}{|c|c|c|c|c|}
\hline $\begin{array}{l}\text { QUANTUM } \\
\text { THEORY }\end{array}$ & $\begin{array}{l}\text { LOCAL HIDDEN } \\
\text { VAR THEORY }\end{array}$ & $\begin{array}{l}\text { LOCAL HIDDEN } \\
\text { VAR THEORY }\end{array}$ & $\begin{array}{l}\text { QUANTUM THY } \\
\text { FEATURES }\end{array}$ & $\begin{array}{l}\text { LHVT } \\
\text { FEATURES }\end{array}$ \\
\hline $\begin{array}{l}\text { Separable } \\
\text { States }\end{array}$ & \multirow{3}{*}{$\begin{array}{l}\text { Bell } \\
\text { Local } \\
\text { States }\end{array}$} & Category 1 & $\begin{array}{l}\text { Quantum } \\
\text { Separable }\end{array}$ & $\begin{array}{l}\text { LHS State } \\
\text { Non-Steer } \\
\text { Bell Local }\end{array}$ \\
\hline \multirow{3}{*}{$\begin{array}{l}\text { Quantum } \\
\text { Entangled } \\
\text { States }\end{array}$} & & Category 2 & $\begin{array}{l}\text { Quantum } \\
\text { Entangled }\end{array}$ & $\begin{array}{l}\text { LHS State } \\
\text { Non-Steer } \\
\text { Bell Local }\end{array}$ \\
\hline & & Category 3 & $\begin{array}{l}\text { Quantum } \\
\text { Entangled }\end{array}$ & $\begin{array}{l}\text { Steerable } \\
\text { Bell Local }\end{array}$ \\
\hline & $\begin{array}{l}\text { Bell Non } \\
\text { Local } \\
\text { States }\end{array}$ & Category 4 & $\begin{array}{l}\text { Quantum } \\
\text { Entangled }\end{array}$ & $\begin{array}{l}\text { Steerable } \\
\text { Bell Non- } \\
\text { Local }\end{array}$ \\
\hline
\end{tabular}

Fig. 1. The Quantum Theory and the Local Hidden Variable Theory Classification Schemes (QTCS and LHVCS). The two categories of quantum states in the QTCS are shown in the left column and the two basic categories of quantum states in the LHVCS are shown in the second left column. The four more detailed categories of quantum states in the LHVCS are shown in the third left column, whilst the right two columns lists the features of the four categories of LHVCS states in both the QTCS and LHVCS schemes.

derived from the basic expression $\sum_{\lambda} P(\lambda, c) P\left(\alpha \mid \Omega_{A}, \lambda, c\right) P\left(\beta \mid \Omega_{B}, \lambda, c\right)$ for the joint probability - is violated in experiment.

\subsection{Two categorizations of states}

Clearly then, the division of the states for bipartite systems into separable and entangled states does not coincide with the categorization of the states into Bell local and Bell non-local. The relationship between these two different schemes is shown in Figure 1. For bipartite systems of identical massive bosons tests for entanglement are set out in $[8,9]$ and tests for EPR steering are presented in [10].

\section{Bell non-locality in microscopic systems}

As pointed out recently [11], there are a multitude of Bell inequalities that can be derived for both multi-partite as well as for bipartite systems, depending on the number of observables considered in each of the sub-systems and on the number of different outcomes for each observable. One of the earliest is the famous $C H S H$ Bell inequality for bipartite systems [12]. Here there were two different observables $\Omega_{A 1}$, $\Omega_{A 2}$ and $\Omega_{B 1}, \Omega_{B 2}$ for each sub-system, and measurement of any observable was restricted to two outcomes - which we choose to be $+1 / 2$ and $-1 / 2$. The CHSH inequality is $|S| \leq 1 / 2$, where $S=\left\langle\Omega_{A 1} \otimes \Omega_{B 1}\right\rangle+\left\langle\Omega_{A 1} \otimes \Omega_{B 2}\right\rangle+\left\langle\Omega_{A 2} \otimes \Omega_{B 1}\right\rangle-$ $\left\langle\Omega_{A 2} \otimes \Omega_{B 2}\right\rangle$. Suitable physical systems for which this inequality can be tested include 
two spin $1 / 2$ sub-systems, with components of the spins along various directions being the observables since the measured outcome is either $+1 / 2$ or $-1 / 2$. Another suitable physical system is two modes of the EM field as the two sub-systems each occupied by one photon, with the mode polarization being the observable - the outcome being $+1 / 2$ or $-1 / 2$ according to whether the outcome is right or left in the case of circular polarization, or up or across in the case of linear polarization. These examples are both microscopic systems. Experiments testing the CHSH inequality in microscopic systems have been carried out since the 1970s (see [11] for a recent review), and a violation of the inequality has now been convincingly demonstrated following numerous improvements to remove possible loopholes via which the inequality might not really be violated.

\section{Macroscopic Bell non-locality}

\subsection{Macroscopic systems}

For systems made up of atoms, a system would be regarded as macroscopic if it contained a very large number of atoms and its overall size scale is large compared to the atomic Bohr radius. Conversely, it would be microscopic if the number of atoms was small and its size was comparable to the Bohr radius. "Macroscopic" is of course only a qualitative term. Note that being macroscopic is not necessarily the same as being describable classically and does not rule out requiring a quantum treatment, though of course a quantum description is needed for microscopic systems. The main point of interest is that if Bell non-locality is exhibited in a macroscopic system, then what Einstein regarded as the strangest feature which distinguishes quantum from classical physics would have occurred in a situation which ought not to require a quantum description. As discussed in the previous section, Bell nonlocality - which requires quantum entanglement (even though this is not sufficient to guarantee Bell non-locality) - has been demonstrated in microscopic systems, but here a classical theory would be expected to fail, so a Bell inequality violation is not so surprising. Bell non-locality in a macroscopic system would be much more unexpected, since this is a regime where a classical theory might be expected to apply. Bell non-locality requires the quantum state to be strongly entangled, and entanglement destroying decoherence effects due to interactions both with the many internal degrees of freedom within a macroscopic system and due to interactions with the external environment could be expected to become more prominent for increasingly macroscopic systems. In comparison, experiments have been carried out with large molecules (regarded as macroscopic systems) that demonstrate quantum interference between two probability amplitudes, even though quantum interference effects might be expected not to occur due to decoherence effects. However, quantum interference is less strange than Bell non-locality because interference also occurs in classical physics. Showing that quantum theory is needed for a macroscopic system is always interesting, and finding Bell locality violations in macroscopic systems would probably represent the most unusual quantum effect that could be found - thus highlighting its importance.

Examples of macroscopic systems in which Bell non-locality could occur include the following. Optical systems involving large photon numbers in entangled field modes have been studied as examples of macroscopic systems even though the notion of system size scale is unclear. A multi-partite system consisting of a very large of microscopic atomic sub-systems (such as in cold atomic gases) in which the atomic sub-systems are entangled is generally be regarded as a potential test bed for macroscopic Bell non-locality. The quantum effect then involves a macroscopic size scale, 
even though the measurement outcomes on the individual sub-systems would be microscopic. On the other hand, a system in which Bell non-locality occurred consisting of just two entangled sub-systems (with each containing only a few modes) would also regarded as demonstrating macroscopic Bell non-locality if large numbers of particles were associated with each sub-system. Although the overall system size scale might not be large, measurement outcomes for each sub-system could have values that are large in terms of units based on Planck's constant and hence lie in the realm where classical physics should apply.

Thus, a more significant (though not a requirement) demonstration of macroscopic Bell non-locality occurs if the physical observables being measured are those whose outcomes are large in units based on Planck's constant rather than only having microscopic outcomes. Bell inequality violations require at least two sub-systems, and although Bell inequalities have been formulated for multi-partite systems [11], finding a Bell inequality violation in bipartite macroscopic systems is preferable for reasons of simplicity as it could involve measurements of a smaller number of observables. A further consideration is that for systems involving identical massive particles such as bosonic or fermionic atoms, where the sub-systems must be defined via distinguishable modes rather than non-distinguishable atoms, the symmetrization principle and the super-selection rules on particle number are recognised as being important in regard to tests for quantum entanglement and EPR steering [8-10]. Hence physically relevant violations of Bell inequalities for both microscopic and macroscopic systems would also only apply for quantum states that comply with the symmetrization principle and the super-selection rules.

\subsection{Ultra-cold atomic gases and Bell tests}

Although proposals for studying Bell non-locality in macroscopic systems have been made since the 1980s involving photonic systems, systems made up of a large number of spin 1/2 particles or systems made up of two high spin particles, the interest in finding Bell non-locality in macroscopic systems has grown during the 2000s (see the review by Reid et al. [13]). This is in part due to experimental progress in the study of ultracold atomic gases, which are macroscopic systems for which a quantum description is required. These include ultracold bosonic gases, where large numbers of bosonic atoms may occupy each mode, creating Bose-Einstein condensates. Measurements based on detecting atom numbers are less error-prone than those involving photon numbers. For studying bipartite Bell non-locality, two mode systems are available such as those for bosons with a single spin state trapped in a double potential well, or for bosons with two different spin states in a single well. A four mode bipartite system involving two modes associated with different internal states in each well can also be prepared [14] using atom-chip techniques. The case treated by Reid et al. [15] (see below) applies to this system. Multipartite systems in which each two state atom is located at a different site on an optical lattice have also been created [16]. For ultracold fermionic gases the situation is not so clear, for although systems with large numbers of fermionic atoms would be macroscopic, each mode could only be occupied by fermions with differing spins, and hence many modes would be involved thus making it difficult to devise bipartite macroscopic systems. In addition to the experimental progress, a range of theoretical approaches have been found for deriving Bell inequalities and a large number of different Bell inequalities have now been obtained. Most only lead to macroscopic non-locality for multi-partite systems, though a few are associated with Bell inequality violations for bipartite systems.

We now review some of the Bell inequalities that have been obtained (presented in historical order) and report on whether experimental tests have been carried out to find violations of the Bell inequality involved. 


\subsection{Mermin (1980), Drummond (1983) Bell inequalities}

There are examples from the 1980s of Bell inequalities applied to macroscopic systems, though no experimental tests have yet been carried out. In reference [17] a system consisting of two large spin $s$ sub-systems was considered allowing for measurements of any spin component to have outcomes from $-s$ to $+s$ in integer steps. For an overall singlet pure state in which measurement of a spin component in one sub-system leads to the opposite outcome when the same spin component was measured in the other, a Bell inequality involving spin components along three unit vectors $a, b, c$ of the form $s\left|\left\langle S_{A a}\right\rangle-\left\langle S_{B b}\right\rangle\right| \geq\left\langle S_{A a} \otimes S_{B c}\right\rangle+\left\langle S_{A b} \otimes S_{B c}\right\rangle$ was found. This was found theoretically to be violated for three distinct coplanar unit vectors, where $a, b$ each make an angle $\pi / 2+\theta$ with $c$ and hence $\pi-2 \theta$ with each other, provided the angle satisfies the condition $0<\sin \theta<1 / 2 s$. This is a very small range of violating angles if $s$ is large enough for the system to be considered macroscopic, and the required singlet state would be difficult to create. Finding particles with large enough $s$ to be macroscopic might possibly be achieved if the "particles": were two mode BEC with large boson numbers prepared in suitable two mode spin states.

In reference [18] two sub-systems each containing two bosonic modes $a_{1}, a_{2}$ or $b_{1}, b_{2}$ was considered. A maximally entangled state of the (un-normalized) form $\left(\widehat{a}_{1}^{\dagger} \widehat{a}_{2}^{\dagger}+\widehat{b}_{1}^{\dagger} \widehat{b}_{2}^{\dagger}\right)^{N}|0\rangle$ with a large number of bosons was studied, and a Bell inequality found involving sub-system boson number-like observables of the form ( $\cos \theta \widehat{b}_{1}^{\dagger}+$ $\left.\sin \theta \widehat{b}_{2}^{\dagger}\right)^{J}\left(\cos \theta \widehat{b}_{1}+\sin \theta \widehat{b}_{2}\right)^{J}$ for sub-system $B$ with mode annihilation operators $\widehat{b}_{1}$, $\widehat{b}_{2}$, with a similar form for sub-system $A$ with mode annihilation operators $\widehat{a}_{1}, \widehat{a}_{2}-$ though here with $\theta=0$. For $J=N \rightarrow \infty$ the inequality is violated for finite $\theta$ if $3 g(\theta)-g(3 \theta)-2>0$, where $g(\theta)=\exp \left(-J \theta^{2} / 2\right)$. Although suitable $\theta$ can be found, the measurement of the observables for large $J=N$ would be difficult, requiring the measurement of a very high order quantum correlation function.

\subsection{MABK (1990-1993) Bell inequalities}

The 1990s saw the introduction [19-21] of the $M A B K$ Bell inequalities to treat multipartite systems with two state spin sub-systems. For GHZ states of the form $(|\uparrow \uparrow \uparrow \uparrow \cdots \uparrow\rangle+i|\downarrow \downarrow \downarrow \downarrow \cdots \downarrow\rangle / \sqrt{2}$ for $n$ sub-systems, a Bell inequality of the form $F \leq 2^{n / 2}$ (even $n$ ) is violated for large $n$, where $F=$ $\operatorname{Im}\left(\sum_{\lambda} P(\lambda)\left\{\begin{array}{c}\left(\left\langle S_{x 1}(\lambda)\right\rangle+i\left\langle S_{y 1}(\lambda)\right\rangle\right) \cdots\left(\left\langle S_{x n}(\lambda)\right\rangle+i\left\langle S_{y n}(\lambda)\right\rangle\right) \\ -\left(\left\langle S_{x 1}(\lambda)\right\rangle-i\left\langle S_{y 1}(\lambda)\right\rangle\right) \cdots\left(\left\langle S_{x n}(\lambda)\right\rangle-i\left\langle S_{y n}(\lambda)\right\rangle\right)\end{array}\right\} / 2 i\right)$ and the spin operators $S_{x i}, S_{y i}$ have outcomes \pm 1 . There is no violation for the bipartite situation $n=2$, which in any case is microscopic. No experimental tests have yet been carried out, and the preparation of the GHZ state would be difficult.

\subsection{Reid et al. (2002) Bell inequalities}

Around 2000 a Bell inequality originally introduced by Clauser et al. [22] was developed by Reid et al. [15] for bipartite systems in which spin observables of the form $S_{Z}^{A}(2 \theta)=S_{z}^{A} \cos 2 \theta+S_{x}^{A} \sin 2 \theta$ and $S_{Z}^{B}(2 \phi)=S_{z}^{B} \cos 2 \phi+S_{x}^{B} \sin 2 \phi$ (both for two mode sub-systems) were measured and their outcomes $-s_{A} / 2, \ldots,+s_{A} / 2$ and $-s_{B} / 2, \ldots,+s_{B} / 2$ divided into positive and negative "bins". Although this would appear to reduce the number of different outcomes to just two for each sub-system, a situation relevant to macroscopic Bell non-locality still appears since the Bell 
inequality is based on considering actual measured outcomes that are large compared to Planck's constant. The joint probabilities $P\left(+,+\mid S_{Z}^{A}(2 \theta), S_{Z}^{B}(2 \phi)\right)$ and the single probabilities $P\left(+\mid S_{Z}^{A}(2 \theta)\right), P\left(+\mid S_{Z}^{B}(2 \phi)\right)$ for positive outcomes then satisfy a Bell inequality of the form $\left\{P(+,+\mid \theta, \phi)-P\left(+,+\mid \theta, \phi^{*}\right)+P\left(+,+\mid \theta^{*}, \phi\right)+\right.$ $\left.P\left(+,+\mid \theta^{*}, \phi^{*}\right)\right\} /\left\{P\left(+\mid \theta^{*}\right)+P(+\mid \phi)\right\} \leq 1$. For the maximally entangled state of the (un-normalized) form $\left(\widehat{a}_{1}^{\dagger} \widehat{a}_{2}^{\dagger}+\widehat{b}_{1}^{\dagger} \widehat{b}_{2}^{\dagger}\right)^{N}|0\rangle \propto \sum_{m=-s}^{s}|s, m\rangle_{A}|s, m\rangle_{B}$ (where here $s_{A}=s_{B}=s=N / 2$ ), Bell inequality violations occurred for a range of parameters $\theta, \theta^{*}, \phi, \phi^{*}$ for both small and large $N$. The large $N$ case corresponds to a macroscopic Bell locality violation in a bipartite system. The original application was to photonic systems, but the theory also applies for ultracold atomic gases. So far, no experimental tests have been made. As in reference [18] the two mode state would be difficult to prepare.

\subsection{Collins et al. (2002) Bell inequalities}

Also in the early 2000s Collins et al. [23] found a different approach (CGLMP) to deriving Bell inequalities. For bipartite systems the treatment assumed the existence of HVT probabilities of the form $P\left(\alpha_{j}, \alpha_{k}, \beta_{l}, \beta_{m} \mid \Omega_{A 1}, \Omega_{A 2}, \Omega_{B 1}, \Omega_{B 2}, c\right)$ (denoted $\left.c_{j, k, l, m}\right)$ for simultaneous measurement outcomes $\alpha_{j}, \alpha_{k}, \beta_{l}, \beta_{m}$ for the pairs of subsystem observables $\Omega_{A 1}, \Omega_{A 2}, \Omega_{B 1}, \Omega_{B 2}$. Clearly, $\sum_{j, k, l, m} c_{j, k, l, m}=1$. The outcomes themselves were the hidden variables, and the hidden variable theory was stated to be local. Although this is not stated, LHVT would require the factorization of the probabilities into $P\left(\alpha_{j}, \alpha_{k} \mid \Omega_{A 1}, \Omega_{A 2}, c\right)$ ( $a_{j, k}$ for short) and $P\left(\beta_{l}, \beta_{m} \mid \Omega_{B 1}, \Omega_{B 2}, c\right)$ ( $b_{l, m}$ for short), thus $c_{j, k, l, m}=a_{j, k} \times b_{l, m}$. The separate sub-system probabilities would satisfy the constraints $\sum_{j, k} a_{j, k}=1$ and $\sum_{l, m} b_{l, m}=1$. The observables for each sub-system were assumed to have the same number of outcomes (listed as $j, k, l, m=0,1, \ldots d-1(\bmod d),-$ thus $\alpha_{d} \equiv \alpha_{0}$, etc.). Probabilities for outcomes for one observable for each sub-system would be obtained as $P\left(\alpha_{j}, \beta_{l} \mid \Omega_{A 1}, \Omega_{B 1}, c\right)=$ $\sum_{k, m} c_{j, k, l, m}=\sum_{k, m} a_{j, k} b_{l, m}$ etc., and probabilities for outcomes for one observable of a specific sub-system given by expressions such as $P\left(\alpha_{j} \mid \Omega_{A 1}, c\right)=\sum_{k, l, m} c_{j, k, l, m}=$ $\sum_{k} a_{j, k}=A 1(j)$ for short.

The idea behind the $C G L M P$ inequalities involves considering joint outcomes for pairs of observables $\Omega_{A}, \Omega_{B}$ for the two sub-systems in which either the outcomes are for the same members of the two outcome lists or where the outcomes refer to different members of the two lists. Probabilities for the same listed outcomes for specific observables for the two sub-systems are given by expressions such as $P\left(\Omega_{A 1}=\Omega_{B 1}\right)=\sum_{j} \sum_{k, m} c_{j, k, j, m}=\sum_{j} \sum_{k, m} a_{j, k} b_{j, m}=\sum_{j} A 1(j) \times B 1(j)$, which is the probability for all outcomes listed $j$ with $\Omega_{A 1}$ leading to $\alpha_{j}$ and all outcomes for $\Omega_{B 1}$ leading to $\beta_{j}$. Probabilities for outcomes for specific observables for the two sub-systems where the listed outcomes are shifted are given by expressions such as $P\left(\Omega_{B 1}=\Omega_{A 2}+1\right)=\sum_{k} \sum_{j, m} c_{j, k,(k+1), m}=\sum_{k} \sum_{j, m} a_{j, k} b_{k+1, m}=$ $\sum_{k} A 2(k) \times B 1(k+1)$, where here we consider all outcomes with $\Omega_{A 2}$ leading to $\alpha_{k}$ and all outcomes for $\Omega_{B 1}$ leading to $\beta_{k+1}$. Combinations of such joint probablities for the four possible pairs of observables $\Omega_{A}, \Omega_{B}$ then involve the basic LHVT probabilities $c_{j, k, l, m}=a_{j, k} \times b_{l, m}$, and are then used to derive Bell inequalities. 
For example, combinations of joint measurement probabilities of the form $I=$ $P\left(\Omega_{A 1}=\Omega_{B 1}\right)+P\left(\Omega_{B 1}=\Omega_{A 2}+1\right)+P\left(\Omega_{A 2}=\Omega_{B 1}\right)+P\left(\Omega_{B 2}=\Omega_{A 1}\right)$ were stated to satisfy $I \leq 3$ for LHVT. Based just on HVT without assuming locality, we have $I=\sum_{j, k, m}\left(c_{j, k, j, m}+c_{j, k,(k+1), m}+c_{j, k, m, k}+c_{j, k, m, j}\right)$. For a given choice of $j, k, m$ there is no reason why all four terms cannot be non-zero (in terms of the notation in Ref. [23], $r^{\prime}+s^{\prime}+t^{\prime}+u^{\prime}=0$ for each term). So as $\sum_{j, k, l, m} c_{j, k, l, m}=1$ and each of the four terms is just a partial contribution to this last equation, it follows that each of the four terms must be between 0 and 1 - since the other part of the contribution also just involves positive terms. Thus, general HVT would imply that $I \leq 4$, as is stated in reference [23]. Also, if the LHVT condition $c_{j, k, l, m}=$ $a_{j, k} \times b_{l, m}$ is invoked we then find that $I=\sum_{j=0}^{d-1}(A 1(j) \cdot B 1(j)+A 2(j) \cdot B 1(j)+$ $A 1(j) \cdot B 2(j))+(A 2(0) \cdot B 1(1)+A 2(1) \cdot B 1(2)+\cdots+A 2(d-2) \cdot B 1(d-1)+A 2(d-$ $1) \cdot B 1(0))$. The individual measurement probabilities $A 1(j), A 2(j), B 1(j), B 2(j)$ are of course all positive and satisfy constraints such as $\sum_{j} A 1(j)=1$, etc. For LHVT it is stated in reference [23] that $I \leq 3$, though no proof is given for this result. However, by multiplying the two constraints for the $A 1(j)$ and the $B 1(j)$, it is easy to establish that $\sum_{j=0}^{d-1} A 1(j) . B 1(j) \leq 1$, since this expression is a partial positive contribution to the overall product of 1 , and the other contribution is also positive. Similar arguments show that $\sum_{j=0}^{d-1} A 2(j) \cdot B 1(j) \leq 1$ and $\sum_{j=0}^{d-1} A 1(j) \cdot B 2(j) \leq 1$. Finally, by multiplying the constraints for $A 2(j)$ and $B 1(j)$ we see that $(A 2(0)$. $B 1(1)+A 2(1) \cdot B 1(2)+\cdots+A 2(d-2) \cdot B 1(d-1)+A 2(d-1) \cdot B 1(0)) \leq 1$, this expression again being a positive partial contribution to the overall product of 1 . Adding together these four inequalities gives $I \leq 4$ for the LHVT case, rather than $I \leq 3$ as stated in reference [23]. A convincing proof of the $I \leq 3$ result for the LHVT case is needed. Hence there is now some doubt as to whether this inequality is a general requirement for LHVT, so its violation does not necessarily show that quantum theory is required to explain the measurements. Other similar expressions to $I$ also led to Bell inequalities, but similar issues apply in these cases as well.

For the (unnormalized) state $\sum_{m=-s}^{s}|s, m\rangle_{A}|s, m\rangle_{B}$ (see above) the quantum expression for $I$ is found to be greater than 3 for all $d=2 s+1$, corresponding to a Bell inequality violation in a macroscopic system if $s=N / 2$ is large. However, this violation involved introducing physical quantities $\Omega_{A 1}, \Omega_{A 2}, \Omega_{B 1}, \Omega_{B 2}$ as Hermitian operators defined by their eigenvalues and eigenvectors (see Eq. (13) in Ref. [23]), the latter being linear combinations of the $|s, m\rangle_{A(B)}$. As the operators turn out to be off-diagonal in these basis states, it is not obvious what physical observable they correspond to. Finally, no experimental tests of the Bell inequalities have been carried out, so for several reasons the Collins et al. [23] inequalities do not look promising as a vehicle for finding macroscopic Bell non-locality.

\subsection{CFRD (2007-2011) Bell inequalities}

Around 2010, a further approach in form of the CFRD Bell inequalities was obtained [24-26]. From the basic LHVT expression (4) for mean values a Bell inequality for bipartite systems with two observables per sub-system is given by $\left\langle\left|\left(\Omega_{A 1}+i \Omega_{A 2}\right)\left(\Omega_{B 1}-i \Omega_{B 2}\right)\right|^{2}\right\rangle \geq\left|\left\langle\left(\Omega_{A 1}+i \Omega_{A 2}\right)\left(\Omega_{B 1}-i \Omega_{B 2}\right)\right\rangle\right|^{2}$ applies. This may also be written as $\left\langle\left(\Omega_{A 1}^{2}+\Omega_{A 2}^{2}\right)\left(\Omega_{B 1}^{2}+\Omega_{B 2}^{2}\right)\right\rangle \geq \mid\left\langle\left(\Omega_{A 1} \Omega_{B 1}+\Omega_{A 2} \Omega_{B 2}\right)\right\rangle+$ $\left.i\left\langle\left(\Omega_{A 2} \Omega_{B 1}-\Omega_{A 1} \Omega_{B 2}\right)\right\rangle\right|^{2}$, where unlike the CHSH inequality a CFRD inequality 
involves both first moment $\left\langle\Omega_{A i} \Omega_{B j}\right\rangle$ and second moment $\left\langle\Omega_{A i}^{2} \Omega_{B j}^{2}\right\rangle$ correlation functions. CFRD type inequalities are also obtained for the multimode case. For bipartite systems each consisting of a single bosonic mode $a$ or $b$ the theory has been applied [25] for the choice of quadrature variables $\Omega_{A 1}=x_{A}, \Omega_{A 2}=p_{A}$ and $\Omega_{B 1}=x_{B}, \Omega_{B 2}=p_{B}$. No Bell inequality violation was found for the GHZ symmetric state $\left(|0\rangle_{A}|1\rangle_{B}+|1\rangle_{A}|0\rangle_{B}\right) / \sqrt{2}-$ which is microscopic anyway. However, by relating the quadrature operators to the two mode spin operators (see Ref. [10]) one can show that to violate the CFRD inequality requires finding a quantum state such that $\left\langle\Delta \widehat{S}_{x}^{2}\right\rangle+\left\langle\Delta \widehat{S}_{y}^{2}\right\rangle+\frac{1}{4}<0$, showing that it can never be violated for any quantum state. For bipartite systems each consisting of two bosonic modes $a_{1}, a_{2}$ or $b_{1}, b_{2}$ the theory has also been applied [26] for the choice of spin variables $\Omega_{A 1}=S_{x}^{A}, \Omega_{A 2}=S_{y}^{A}$ and $\Omega_{B 1}=S_{x}^{B}, \Omega_{B 2}=S_{y}^{B}$. For the (unnormalized) quantum state $\sum_{m=-s}^{s} r_{m}|s, m\rangle_{A}|s, m\rangle_{B}$ (where the $r_{m}$ were chosen to optimize the non-locality condition), no violation of the Bell inequality was found except for the case $s=\frac{1}{2}$, corresponding to one boson in each subsystem - a microscopic case. Other choices of observables such as $\Omega_{A 1}=\underline{S}_{\rightarrow}^{A} \cdot \underset{u_{1}}{\rightarrow}, \Omega_{A 2}=\underline{S}_{\rightarrow}^{A} \cdot \underline{u}_{2}, \Omega_{B 1}=\underline{S}_{\rightarrow}^{B} \cdot \underline{v_{1}}$, $\Omega_{B 2}=\underline{S}^{B} \cdot \underline{v}_{2}$ and other choices of quantum state could perhaps result in a Bell inequality violation - however such cases are yet to be explored. When applied to multi-partite situations, the CFRD inequalities do lead to Bell inequality violations for any spin $s$ when the numbers of sub-systems becomes large enough (see Fig. 6 in Ref. [26]). The reason for this effect is still not understood. So far, no experimental tests have been made.

\subsection{Tura et al. (2014) Bell inequalities}

More recent discussions of Bell non-locality in many-body systems are presented in references [11,27-29], based on treating the allowed LHVT probabilities in terms of the theory of polytopes These contain examples of multipartite Bell inequalities, with applications to systems such as two state atoms located at different sites in an optical lattice. Here each identical atom $i=1, \ldots, N$ is treated as a distinguishable two mode pseudo-spin sub-system. Measurements on one of two chosen spin components $M_{i 0}$ or $M_{i 1}$ for the $i$ th atom sub-system are considered, the two possible outcomes being designated as $\alpha_{i}= \pm 1$. Defining $S_{0}=\sum_{i}\left\langle M_{i 0}\right\rangle, S_{00}=\sum_{i, j(i \neq j)}\left\langle M_{i 0} M_{j 0}\right\rangle$, $S_{11}=\sum_{i, j(i \neq j)}\left\langle M_{i 1} M_{j 1}\right\rangle$ and $S_{01}=\sum_{i, j(i \neq j)}\left\langle M_{i 0} M_{j 1}\right\rangle$ involving the mean values of single measurements on individual spins or joint measurements on different spins, a Bell inequality $2 S_{0}+S_{01}+2 N+\left(S_{00}+S_{11}\right) / 2 \geq 0$ has been derived [27]. Bell inequality violations were predicted for Dicke states [30]. These have the advantage of being the lowest energy eigenstates for certain many-body Hamiltonians that describe physical systems, such as $N$ spins interacting via two-body ferromagnetic coupling, so experimental situations to search for Bell inequality violations were seen as being readily available. Indeed, Bell correlations based on this inequality have actually been found $[28,29]$ in systems involving $5 \times 10^{2}$ and $5 \times 10^{5}$ bosonic atoms prepared in spin squeezed states. Two component bosonic atoms were localised on optical or magnetic lattices, with the two spin states being coupled via Rabi fields. Spin squeezing occurred due to inter-atomic collisions. In these systems the indistinguishability of the identical atoms and the effect of super-selection rules that rule out sub-system states with coherences between different boson numbers was ignored, as there is just one atom in each separated spatial mode on each different lattice site. However, there is no macroscopic violation of Bell locality in the bipartite case, since this would only 
correspond to just two atoms. Nevertheless, these two experiments provide examples of Bell non-locality in a macroscopic system, albeit for the multi-partite situation.

\subsection{Dalton (2017) - generalised CHSH Bell inequality}

Finally, a more standard application of LHVT for bipartite systems each with two bosonic modes and involving spin observables, leads to the following Bell inequality $-|S| \leq \frac{1}{2}\left\langle N_{A}\right\rangle\left\langle N_{B}\right\rangle$, where $S=\left\langle\Omega_{A 1} \otimes \Omega_{B 1}\right\rangle+\left\langle\Omega_{A 1} \otimes \Omega_{B 2}\right\rangle+\left\langle\Omega_{A 2} \otimes \Omega_{B 1}\right\rangle-$ $\left\langle\Omega_{A 2} \otimes \Omega_{B 2}\right\rangle$ and $\Omega_{A 1}=\underline{S}_{\rightarrow}^{A} \cdot \underline{u_{1}}, \Omega_{A 2}=\underline{S}_{\rightarrow}^{A} \cdot \underset{u_{2}}{\rightarrow}, \Omega_{B 1}=\underline{S}_{\rightarrow}^{B} \cdot \underline{v_{1}}, \Omega_{B 2}=\underline{S}_{\rightarrow}^{B} \cdot \underline{v_{2}}$ are components of the spin observables, with $N_{A}, \overrightarrow{N_{B}}$ giving the number of bosons in each sub-system. This inequality is a generalisation of the CHSH inequality and its derivation is similar. Details are given in reference [10] (see version 1, Sect. 6.1). For the case of spin $\frac{1}{2}$ sub-systems this reduces to the CHSH inequality. Several different quantum states have been tested for violation of this Bell inequality. These included (a) the relative phase eigenstate $\sum_{k=-n / 2}^{+n / 2} \exp (i k \theta)\left|\frac{n}{2}, k\right\rangle_{A}\left|\frac{n}{2},-k\right\rangle_{B} / \sqrt{n+1}$ [31], (b) the maximally entangled state $\sum_{k=-n / 2}^{+n / 2}\left|\frac{n}{2}, k\right\rangle_{A}\left|\frac{n}{2}, k\right\rangle_{B} / \sqrt{n+1}$, (c) the Werner [6] states $\widehat{\rho}_{W}=\left(d^{3}-d\right)^{-1}((d-\phi) \widehat{1}+(d \phi-1) \widehat{V})$, (where $d=n+1$ and $\hat{1}$ is the unit operator defined in the $d \times d$ space whose basis vectors are $\left|\frac{n}{2}, k\right\rangle_{A}\left|\frac{n}{2}, l\right\rangle_{B}$ with $k, l=$ $-n / 2,-n / 2+1, \ldots,+n / 2$, and $\widehat{V}$ is the flip operator defined by $\widehat{V}\left|\frac{n}{2}, k\right\rangle_{A}\left|\frac{n}{2}, l\right\rangle_{B}=$ $\left|\frac{n}{2}, l\right\rangle_{A}\left|\frac{n}{2}, k\right\rangle_{B}$. Physical restrictions on the parameter $\phi$ are $+1 \geq \phi \geq-1$ ) and (d) the angular momentum eigenstates

$$
\sum_{k_{A}, k_{B}} C\left(\frac{N_{A}}{2}, \frac{N_{B}}{2}, J ; k_{A}, k_{B}, K\right)\left|\frac{N_{A}}{2}, k_{A}\right\rangle_{A}\left|\frac{N_{B}}{2}, k_{B}\right\rangle_{B}
$$

where the $C\left(\frac{N_{A}}{2}, \frac{N_{B}}{2}, J ; k_{A}, k_{B}, K\right)$ are Clebsch-Gordon coefficients. Numerical optimization methods to choose the four spin components were used. For these four cases the Bell inequality was only violated occurred for the microscopic case where $N_{A}=$ $N_{B}=1$, which just corresponds to the CHSH situation. Other states, such as spin squeezed states would be worth studying. As the mean values $\left\langle\underline{S}_{\rightarrow}^{A} \cdot \underline{u}_{\rightarrow} \otimes \underline{S}_{\rightarrow}^{B} \cdot \underline{v}_{\rightarrow}\right\rangle$ for products of these spin operators for bipartite systems (each containing two modes) can be measured fairly easily using mode couplers with suitable phases and pulse lengths, then finding a suitable quantum state with large $\left\langle N_{A}\right\rangle$ and $\left\langle N_{B}\right\rangle$ where the Bell inequality was violated would provide a case of macroscopic Bell non-locality.

\section{Conclusions}

A number of different forms of Bell inequalities have been obtained over the last four decades, which could be tested to find Bell non-locality in macroscopic systems. A successful outcome would be highly significant, establishing the priority of the Copenhagen quantum theory over local hidden variable theories for systems where a classically based theory might be expected to apply. Up to the present, only two experiments $[28,29]$ have achieved this, based on the versions of Bell inequalities derived by Tura et al. [27]. These experiments however are for the multi-partite situation rather than the simpler bipartite case. The derivation of testable Bell inequalities for macroscopic bipartite (rather than multi-partite) systems is an ongoing issue, as 
is the experimental search for more cases of macroscopic Bell non-locality. As quantum states that demonstrate Bell non-locality involve strong entanglement, the issue of preparing states for which entanglement-destroying decoherence effects are minimised will be important, since these effects tend to be more significant in macroscopic systems.

The author wishes to acknowledge discussions with S.M. Barnett, M.D. Reid, J.A. Vaccaro and H.M. Wiseman, and thanks the referees for their helpful comments. BJD also thanks the Centre for Cold Matter, Imperial College, London for its hospitality during the writing of this article.

Note added in proof. A proof of the CGLMP inequality $I \leq 3$ (see Sect. 4.6) is provided in a forthcoming paper [32]. However $I \leq 3$ is shown to apply for all hidden variable theories, both local and non-local. That $I \leq 4$ applies for all HVT is then trivially true.

\section{Author contribution statement}

The paper was entirely written by B.J. Dalton.

Open Access This is an open access article distributed under the terms of the Creative Commons Attribution License (http://creativecommons.org/licenses/by/4.0), which permits unrestricted use, distribution, and reproduction in any medium, provided the original work is properly cited.

\section{References}

1. A. Einstein, B. Podolsky, N. Rosen, Phys. Rev. 47, 777 (1935)

2. E. Schrodinger, Proc. Camb. Phil. Soc. 31, 553 (1935)

3. J.S. Bell, Physics 1, 195 (1964)

4. B.S. DeWitt, The Many Worlds Interpretation of Quantum Mechanics (Princeton University Press, 1973)

5. D. Bohm, Phys. Rev. 85, 166 (1952)

6. R.F. Werner, Phys. Rev. A 40, 4277 (1989)

7. H.M. Wiseman, S.J. Jones, A.C. Doherty, Phys. Rev. Lett. 98, 140402 (2007)

8. B.J. Dalton, J. Goold, B.M. Garraway, M.D. Reid, Phys. Scr. 92, 023004 (2017)

9. B.J. Dalton, J. Goold, B.M. Garraway, M.D. Reid, Phys. Scr. 92, 023005 (2017)

10. B.J. Dalton, M.D. Reid, arXiv:1611.09101 [qu-ph] (2017)

11. N. Brunner, D. Cavalcanti, S. Pironio, V. Scarani, S. Werner, Rev. Mod. Phys. 86, 419 (2014)

12. J.F. Clauser, M.A. Horne, A. Shimony, R.A. Holt, Phys. Rev. Lett. 23, 880 (1969)

13. M.D. Reid, Q.-Y. He, P.D. Drummond, Front. Phys. 7, 72 (2012)

14. M.F. Riedel, P. Bohi, Y. Li, T. W. Hansch, A. Sinatra, P. Treutlein, Nature 464, 1170 (2010)

15. M.D. Reid, W.J. Munro, F. De Martini, Phys. Rev. A 66, 033801 (2002)

16. C. Gross, T. Zibold, E. Nicklas, J. Esteve, M.K. Oberthaler, Nature 464, 1165 (2010)

17. N.D. Mermin, Phys. Rev. D 22, 356 (1980)

18. P.D. Drummond, Phys. Rev. Lett. 50, 407 (1983)

19. N.D. Mermin, Phys. Rev. Lett. 65, 1838 (1990)

20. M. Ardehali, Phys. Rev. A 46, 5375 (1992)

21. A.V. Belinskii, D.N. Klyshko, Phys. Usp. 36, 654 (1993)

22. J.F. Clauser, A. Shimony, Rep. Prog. Phys. 41, 1881 (1978) 
23. D. Collins, N. Gisin, N. Linden, S. Massar, S. Popescu, Phys. Rev. Lett. 88, 040404 (2002)

24. E.G. Cavalcanti, C.J. Foster, M.D. Reid, P.D. Drummond, Phys. Rev. Lett. 99, 210405 (2007)

25. Q.Y. He, E.G. Cavalcanti, M.D. Reid, P.D. Drummond, Phys. Rev. A 81, 062106 (2010)

26. Q.Y. He, P.D. Drummond, M.D. Reid, Phys. Rev. A 83, 032120 (2011)

27. J. Tura, R. Augusiak, A.B. Sainz, T. Vertesi, M. Lewenstein, A. Acin, Science 344, 1256 (2014)

28. R. Schmied, J.-D. Bancal, B. Allard, M. Fadel, V. Scarani, P. Treutlein, N. Sangouard, Science 352, 441 (2016)

29. N.J. Engelsen, R. Krishnakumar, O. Hosten, M.A. Kasevich, Phys. Rev. Lett. 118, $140401(2017)$

30. R.H. Dicke, Phys. Rev. 93, 99 (1954)

31. B.J. Dalton, S. Ghanbari, J. Mod. Opt. 59, 287 (2012)

32. B.J. Dalton, The CGLMP Bell inequalities and quantum theory, arXiv:1812.09651 (2018) 